\title{
Dual boundary element analysis of wave scattering from singularities
}

\author{
J.T. Chen ${ }^{\mathrm{a}, *}$, M.T. Liang ${ }^{\text {a }}$, I.L. Chen ${ }^{\text {a }}$, S.W. Chyuan ${ }^{\text {b }}$, K.H. Chen ${ }^{\text {a }}$ \\ ${ }^{a}$ Department of Harbor and River Engineering, National Taiwan Ocean University, P.O. Box 7-59, Keelung 202, Taiwan \\ ${ }^{\mathrm{b}}$ Chungshan Institute of Science and Technology, Lungtan, Taiwan \\ Received 7 May 1998; received in revised form 29 January 1999; accepted 18 February 1999
}

\begin{abstract}
The dual boundary element method is used to obtain an efficient solution of the Helmholtz equation in the presence of geometric singularities. In particular, time-harmonic waves in a membrane which contains one or more fixed edge stringers (or cracks) are investigated. The hypersingular integral equation is used in the procedure to ensure a unique solution for the problem with a degenerate boundary. The method yields a solution for the entire membrane as well as the dynamic stress intensity factor. Numerical results are presented for a circular membrane containing a single edge stringer, two edge stringers and an internal stringer. Also, the first three critical wave numbers of the membrane with the homogeneous boundary condition are determined, and the dynamic stress intensity factors are found for problems with the nonhomogeneous boundary condition. Good agreement is found after comparing the results with exact solutions, and with results obtained using DtN-FEM and ABAQUS. (C1999 Elsevier Science B.V. All rights reserved.
\end{abstract}

\section{Introduction}

Analytical solutions for the stresses around cracks, even in the static case, are available only in very simple cases, primarily for problems in the infinite domain. Exact solutions are even more scarce. Therefore, numerical treatment of such problems is necessary to obtain solutions, e.g., using the finite difference method, finite element method or boundary element method.

In studying the membrane problem with stringers using numerical techniques, the analyst may encounter problems with singularities. Singular behavior is often ignored based on the expectation that the error will be limited to the vicinity of the singularity. However, it is very important to show how strong the singular behavior is, e.g., the stress intensity factor of fracture mechanics. In finite elements, special singular or hybrid elements are sometimes used instead of the quarter-point rule; e.g., MSC/NASTRAN Version 68 [1] provides the capabilities of singular CRAC3D and CRAC2D elements for static crack problems, but dynamic cases with singularities for the Helmholtz equation have not been developed to the authors' best knowledge.

\footnotetext{
* Corresponding author. Fax: +886-2-462-2192-6102

E-mail address: b0209@ntou66.ntou.edu.tw (J.T. Chen)
} 


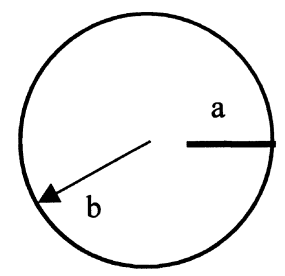

(a)



(b)

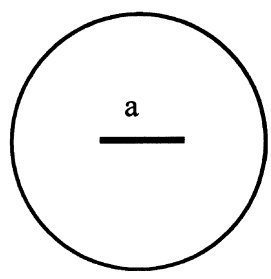

(c)

Fig. 1. A vibrating membrane: (a) with a single edge stringer, (b) with two opposite edge stringers, (c) with an internal stringer.

For the problems with a degenerate boundary, some special techniques have been devised to solve the difficulty. In crack problems, the special Green's function methods [2] and zone method [3] are worth mentioning. The special Green's function, which satisfies the boundary conditions on the crack, is obtainable. The zone method introduces an artificial boundary in the intact area to connect cracks and the boundary and thus divides the domain into zones by employing a superelement concept. The drawback is obvious in that the artificial boundary is arbitrary, not unique, and thus not qualified as an automatic scheme. For problems with a degenerate boundary, e.g., crack problems [4], flow around sheet piles [5], thin airfoil in aerodynamics [6,7], cracked bars under torsion [8], and acoustic modes in a cavity with an incomplete partition [9-12], a singularity exists, and the dual integral formulation has been applied successfully. Using the dual integral formulation, well-posed boundary value problems can be solved even though a degenerate boundary is present. It is well known that DBEM (dual boundary element method) is particularly suitable for problems of extreme localization and concentration with a singularity [13]. The main difference between the present method and the Burton-Miller method [14] is that the Burton-Miller method combines the singular and hypersingular equations by an imaginary constant to avoid the fictitious eigenvalues in the exterior problem. The present dual BEM utilizes the two equations (singular and hypersingular) at the same time. Dual BEM has been developed to solve crack problems (static or dynamic) [15-18]. Also, a single-domain approach by discretizing the boundary only using dual BEM can be achieved instead of the method of subdomains by introducing an artificial boundary $[19,20]$. The DBEM solution is based on a complete formulation of dual integral equations. The long standing abstruseness of the nonuniqueness problem in BEM has been solved, and the general purpose program of BEPO2D (Boundary Element POtential 2-D) has been implemented [7] to solve some potential problems.

In this paper, the dual BEM is extended to solve a problem of a two-dimensional membrane with stringers. The stringer boundary is degenerate since it has infinite stiffness which enforces a displacement constraint. A general purpose program, DUALHAK, is implemented to determine the critical wave numbers and the dynamic stress intensity factors. Numerical experiments for a single edge stringer, two edge stringers and an internal stringer have been carried out to check the validity of the present formulation. The results have been compared with analytical solutions and with solutions obtained using DtN-FEM developed by Givoli and Vigdergauz [21] and ABAQUS to determine their accuracy. Also, the critical wave numbers for stringers of different lengths have been determined.

\section{Dual integral formulation for a membrane problem with degenerate boundaries of stringers}

Consider a membrane problem, as shown in Fig. 1, which has the following governing equation:

$$
\nabla^{2} \phi(x)+k^{2} \phi(x)=0, \quad x \text { in } D,
$$

where $D$ is the domain of interest, $x$ is the domain point, $\phi$ is the displacement and $k$ is the wave number. The boundary conditions are given as follows:

$$
\begin{aligned}
& \phi(x)=\bar{\phi}, \quad x \text { on } B_{1}, \\
& \frac{\partial \phi(x)}{\partial n_{x}}=\bar{t}, \quad x \text { on } B_{2},
\end{aligned}
$$


Table 1

The explicit form of kernel functions for the two-dimensional Helmholtz equation ${ }^{\mathrm{a}}$

\begin{tabular}{lllll}
\hline Kernel function & $U(s, x)$ & $T(s, x)$ & $L(s, x)$ & $M(s, x)$ \\
Order of & weak & strong & strong & hypersingular \\
$\quad$ singularity & $O(\ln (r))$ & $O(1 / r)$ & $O(1 / r)$ & $O\left(1 / r^{2}\right)$ \\
Symmetry & $U(x, s)$ & $L(x, s)$ & $T(x, s)$ & $M(x, s)$ \\
Two dimensional & $-i \pi H_{0}^{(1)}(k r) / 2$ & $(-i k \pi / 2) H_{1}^{(1)}(k r)$ & $(i k \pi / 2) H_{1}^{(1)}(k r)$ & $(-i k \pi / 2)\left\{-k\left(H_{2}^{(1)}(k r) / r^{2}\right) y_{i} y_{j} n_{i} \bar{n}_{j}\right.$ \\
$\quad$ case & & $\times\left(y_{i} n_{i} / r\right)$ & $\times\left(y_{i} \bar{n}_{i} / r\right)$ & $\left.+\left(H_{1}^{(1)}(k r) / r\right) n_{i} \bar{n}_{i}\right\}$ \\
Remark & $r^{2}=y_{i} y_{i}$ & $n_{i}=n_{i}(s)$ & $\bar{n}_{i}=n_{i}(x)$ & $y_{i}=x_{i}-s_{i}$ \\
\hline
\end{tabular}

${ }^{\mathrm{a}} H_{m}^{(1)}(k r)$ denotes the first kind of $m$ th order Hankel function.

where $B_{1}$ is the essential boundary with specified displacement, $B_{2}$ is the natural boundary where the normal derivative of the displacement in the $n_{x}$ direction is specified, and $B_{1}$ and $B_{2}$ comprise the whole boundary of the domain $D$. For the homogeneous boundary conditions of $\bar{\phi}=0$ and $\bar{t}=0$, we have an eigenproblem to determine the critical wave number $k$. For the nonhomogeneous boundary conditions and a specified $k$ value, we can solve for the time-harmonic response and calculate the dynamic stress intensity factors.

The first equation of the dual boundary integral equations for the domain point can be derived from Green's third identity:

$$
2 \pi \phi(x)=\int_{B} T(s, x) \phi(s) \mathrm{d} B(s)-\int_{B} U(s, x) \frac{\partial \phi(s)}{\partial n_{s}} \mathrm{~d} B(s), \quad x \in D,
$$

where $U(x, s)$ is the fundamental solution which satisfies

$$
\nabla^{2} U(x, s)+k^{2} U(x, s)=\delta(x-s), \quad x \in D,
$$

in which $\delta(x-s)$ is the Dirac-delta function, and $T(s, x)$ is defined by

$$
T(s, x) \equiv \frac{\partial U(s, x)}{\partial n_{s}},
$$

in which $n_{s}$ is the outward directed normal at the boundary point $s$. Since a stringer is present in the membrane, one alternative way to solve the problem with a degenerate boundary is to derive the hypersingular equation. Although the hypersingular equation is not absolutely necessary for solving the problem, special considerations [3] or special functions [2] to satisfy the boundary condition on the stringer are needed. After taking the normal derivative with respect to Eq. (4), the second equation of the dual boundary integral equations for the domain point can be derived:

$$
2 \pi \frac{\partial \phi(x)}{\partial n_{x}}=\int_{B} M(s, x) \phi(s) \mathrm{d} B(s)-\int_{B} L(s, x) \frac{\partial \phi(s)}{\partial n_{s}} \mathrm{~d} B(s), \quad x \in D,
$$

where

$$
\begin{aligned}
& L(s, x) \equiv \frac{\partial U(s, x)}{\partial n_{x}}, \\
& M(s, x) \equiv \frac{\partial^{2} U(s, x)}{\partial n_{x} \partial n_{s}},
\end{aligned}
$$

in which $n_{x}$ and $n_{s}$ represent the outward directed normals of $x$ and $s$, respectively. The explicit forms for the four kernel functions are shown in Table 1. By moving the field point $x$ in Eqs. (4) and (7) to the boundary, the dual boundary integral equations for the boundary point can be obtained as follows:

$$
\pi \phi(x)=\text { C.P.V. } \int_{B} T(s, x) \phi(s) \mathrm{d} B(s)-\text { R.P.V. } \int_{B} U(s, x) \frac{\partial \phi(s)}{\partial n_{s}} \mathrm{~d} B(s), \quad x \in B,
$$




$$
\pi \frac{\partial \phi(x)}{\partial n_{x}}=\text { H.P.V. } \int_{B} M(s, x) \phi(s) \mathrm{d} B(s)-\text { C.P.V. } \int_{B} L(s, x) \frac{\partial \phi(s)}{\partial n_{s}} \mathrm{~d} B(s), \quad x \in B,
$$

where R.P.V. is the Riemann principal value, C.P.V. is the Cauchy principal value and H.P.V. is the Hadamard (Mangler) principal value.

It must be noted that Eq. (11) can be derived directly by applying a normal derivative operator with respect to Eq. (10). Differentiation of the Cauchy principal value should be carried out carefully using Leibnitz's rule. The commutative property provides us with two alternatives for calculating the Hadamard principal value in the same way as used for crack problems [4]. For the problem including a normal boundary $S$ and degenerate boundaries $C^{+}+C^{-}$, i.e., $B=S+C^{+}+C^{-}$, Eqs. (10) and (11) can be reformulated as follows: When $x$ is located on the normal boundary, i.e., $x \in S$, Eqs. (10) and (11) become

$$
\begin{aligned}
\pi \phi(x)= & \text { C.P.V. } \int_{S} T(s, x) \phi(s) \mathrm{d} B(s)-\text { R.P.V. } \int_{S} U(s, x) \frac{\partial \phi(s)}{\partial n_{s}} \mathrm{~d} B(s)+\int_{C^{+}} T(s, x) \Delta \phi(s) \mathrm{d} B(s) \\
& -\int_{C^{+}} U(s, x) \Sigma \frac{\partial \phi(s)}{\partial n_{s}} \mathrm{~d} B(s), \\
\pi \frac{\partial \phi(x)}{\partial n_{x}}= & \text { H.P.V. } \int_{S} M(s, x) \phi(s) \mathrm{d} B(s)-\text { C.P.V. } \int_{S} L(s, x) \frac{\partial \phi(s)}{\partial n_{s}} \mathrm{~d} B(s)+\int_{C^{+}} M(s, x) \Delta \phi(s) \mathrm{d} B(s) \\
& -\int_{C^{+}} L(s, x) \Sigma \frac{\partial \phi(s)}{\partial n_{s}} \mathrm{~d} B(s),
\end{aligned}
$$

where

$$
\begin{aligned}
& \Delta \phi(s)=\phi\left(s^{+}\right)-\phi\left(s^{-}\right), \\
& \Sigma \frac{\partial \phi}{\partial n}(s)=\frac{\partial \phi}{\partial n}\left(s^{+}\right)+\frac{\partial \phi}{\partial n}\left(s^{-}\right) .
\end{aligned}
$$

When $x$ is located on the boundary of the stringer, i.e., $x \in C^{+}$, Eqs. (10) and (11) reduce to

$$
\begin{aligned}
\pi \Sigma \phi(x)= & \text { C.P.V. } \int_{C^{+}} T(s, x) \Delta \phi(s) \mathrm{d} B(s)-\text { R.P.V. } \int_{C^{+}} U(s, x) \Sigma \frac{\partial \phi(s)}{\partial n_{s}} \mathrm{~d} B(s)+\int_{S} T(s, x) \phi(s) \mathrm{d} B(s) \\
& -\int_{S} U(s, x) \frac{\partial \phi(s)}{\partial n_{s}} \mathrm{~d} B(s), \\
\pi \Delta \frac{\partial \phi(x)}{\partial n_{x}}= & \text { H.P.V. } \int_{C^{+}} M(s, x) \Delta \phi(s) \mathrm{d} B(s)-\text { C.P.V. } \int_{C^{+}} L(s, x) \Sigma \frac{\partial \phi(s)}{\partial n_{s}} \mathrm{~d} B(s) \\
& +\int_{S} M(s, x) \phi(s) \mathrm{d} B(s)-\int_{S} L(s, x) \frac{\partial \phi(s)}{\partial n_{s}} \mathrm{~d} B(s),
\end{aligned}
$$

where

$$
\begin{aligned}
& \Sigma \phi(x)=\phi\left(x^{+}\right)+\phi\left(x^{-}\right), \\
& \Delta \frac{\partial \phi}{\partial n}(x)=\frac{\partial \phi}{\partial n}\left(x^{+}\right)-\frac{\partial \phi}{\partial n}\left(x^{-}\right) .
\end{aligned}
$$

For the fixed stringer problem, the term of $\Delta \phi$ and $\Sigma \phi$ both disappear. For the traction-free crack problem, $\Delta \partial \phi / \partial n$ and $\Sigma \partial \phi / \partial n$ both disappear [4]. Eqs. (14),(15),(18) and (19) indicate that the unknowns on the degenerate boundary double, and that the additional hypersingular integral equation, Eq. (17), is correspondingly necessary; i.e., the dual boundary integral equations can provide us with sufficient constraint relations for the doubled boundary unknowns on the degenerate boundary. 


\section{Dual boundary element formulation using the constant element scheme}

After deriving the above compatible relationship among the boundary data in Eqs. (10) and (11), the dual boundary integral equations can be discretized by using $N$ constant elements, and the resulting algebraic system can be obtained as

$$
\begin{aligned}
& {\left[\bar{T}_{i j}\right]\{\phi\}=\left[U_{i j}\right]\left\{\frac{\partial \phi}{\partial n}\right\},} \\
& {\left[M_{i j}\right]\{\phi\}=\left[\bar{L}_{i j}\right]\left\{\frac{\partial \phi}{\partial n}\right\},}
\end{aligned}
$$

where [ ] denotes a square matrix with dimension $N$ by $N,\{\}$ is a column vector for the boundary data and the elements of the square matrices are, respectively,

$$
\begin{aligned}
& U_{i j}=\text { R.P.V. } \int_{B_{j}} U\left(s_{j}, x_{i}\right) \mathrm{d} B\left(s_{j}\right), \\
& \bar{T}_{i j}=-\pi \delta_{i j}+\text { C.P.V. } \int_{B_{j}} T\left(s_{j}, x_{i}\right) \mathrm{d} B\left(s_{j}\right), \\
& \bar{L}_{i j}=\pi \delta_{i j}+\text { C.P.V. } \int_{B_{j}} L\left(s_{j}, x_{i}\right) \mathrm{d} B\left(s_{j}\right), \\
& M_{i j}=\text { H.P.V. } \int_{B_{j}} M\left(s_{j}, x_{i}\right) \mathrm{d} B\left(s_{j}\right),
\end{aligned}
$$

where $B_{j}$ denotes the $j$ th element and $\delta_{i j}$ is the Kronecker delta. All the above formulas can be separated into two parts: one is regular and the other is irregular. For the irregular part, partial integration is employed to transform the hypersingular, strongly singular and weakly singular integrals into regular integrations. Therefore, the quadrature rule can be used to determine all the integrals.

Since the terms involving the irregular integrals are the diagonal elements of the four matrices in Eqs. (22)-(25), we have

1. $U(s, x)$ kernel:

$$
\begin{aligned}
U_{\mathbf{i i}} & =\frac{-i \pi}{2} \lim _{\epsilon \rightarrow 0} \int_{-0.5 l}^{0.5 l} H_{0}^{(1)}\left(k \sqrt{s^{2}+\epsilon^{2}}\right) \mathrm{d} s \\
& =\frac{-i \pi}{2} \lim _{\epsilon \rightarrow 0}\left\{\int_{-0.5 l}^{-\sqrt{\epsilon}} H_{0}^{(1)}(k|s|) \mathrm{d} s+\int_{-\sqrt{\epsilon}}^{\sqrt{\epsilon}} i \frac{2}{\pi} \ln \left(\frac{k}{2} \sqrt{s^{2}+\epsilon^{2}}\right) \mathrm{d} s+\int_{\sqrt{\epsilon}}^{0.5 l} H_{0}^{(1)}(k s) \mathrm{d} s\right\} \\
& =\frac{-i \pi}{2} \lim _{\epsilon \rightarrow 0}\left\{\int_{-0.5 l}^{-\sqrt{\epsilon}} H_{0}^{(1)}(k|s|) \mathrm{d} s+0+\int_{\sqrt{\epsilon}}^{0.5 l} H_{0}^{(1)}(k s) \mathrm{d} s\right\} \\
& =\frac{-i \pi}{2}\left\{H_{0}^{(1)}\left(\frac{k l}{2}\right) l+k \int_{-0.5 l}^{0.5 l}\left\{H_{1}^{(1)}(k|s|)|s| \mathrm{d} s\right\}\right\} \text { (i no sum); }
\end{aligned}
$$

2. $T(s, x)$ kernel:

$$
\begin{aligned}
T_{\mathbf{i i}} & =\frac{i \pi k}{2} \lim _{\epsilon \rightarrow 0} \int_{-0.5 l}^{0.5 l} H_{1}^{(1)}\left(k \sqrt{s^{2}+\epsilon^{2}}\right) \frac{\epsilon}{\sqrt{s^{2}+\epsilon^{2}}} \mathrm{~d} s=\frac{i \pi k}{2} \lim _{\epsilon \rightarrow 0} \int_{-\sqrt[4]{\epsilon}}^{\sqrt[4]{\epsilon}} \frac{i(-2)}{\pi k \sqrt{s^{2}+\epsilon^{2}}} \frac{\epsilon}{\sqrt{s^{2}+\epsilon^{2}}} \mathrm{~d} s \\
& =\left.\lim _{\epsilon \rightarrow 0} \arctan \frac{s}{\epsilon}\right|_{-\sqrt[4]{\epsilon}} ^{\sqrt[4]{\epsilon}}=\pi(\mathbf{i} \text { no sum }) ;
\end{aligned}
$$


3. $L(s, x)$ kernel:

$$
\begin{aligned}
L_{\mathrm{ii}} & =\frac{i \pi k}{2} \lim _{\epsilon \rightarrow 0} \int_{-0.5 l}^{0.5 l} H_{1}^{(1)}\left(k \sqrt{s^{2}+\epsilon^{2}}\right) \frac{-\epsilon}{\sqrt{s^{2}+\epsilon^{2}}} \mathrm{~d} s \\
& =\lim _{\epsilon \rightarrow 0} \frac{-i \pi k}{2} \int_{-\sqrt[4]{\epsilon}}^{\sqrt[4]{\epsilon}} \frac{i(-2)}{\pi k \sqrt{s^{2}+\epsilon^{2}}} \frac{\epsilon}{\sqrt{s^{2}+\epsilon^{2}}} \mathrm{~d} s=-\pi \text { (i no sum); }
\end{aligned}
$$

4. $M(s, x)$ kernel:

$$
\begin{aligned}
M_{\mathbf{i i}} & =\frac{-i \pi k}{2} \lim _{\epsilon \rightarrow 0} \int_{-0.5 l}^{0.5 l}\left\{-k \frac{H_{2}^{(1)}\left(k \sqrt{s^{2}+\epsilon^{2}}\right)}{s^{2}+\epsilon^{2}}(-\epsilon)(-\epsilon)+\frac{H_{1}^{(1)}\left(k \sqrt{s^{2}+\epsilon^{2}}\right)}{\sqrt{s^{2}+\epsilon^{2}}}\right\} \mathrm{d} s \\
& =\frac{-i \pi k}{2}\left\{-2 H_{1}^{(1)}\left(\frac{k l}{2}\right)+k\left[H_{0}^{(1)}\left(\frac{k l}{2}\right)+k \int_{-0.5 l}^{0.5 l} H_{1}^{(1)}(k|s|)|s| \mathrm{d} s\right]\right\} \text { (i no sum). }
\end{aligned}
$$

\section{Eigenequation for the membrane with stringers}

For the membrane with stringers, the Dirichlet problem of the homogeneous boundary condition is considered here. After determining the influence coefficients and substituting the boundary conditions, we can obtain the transcendental eigenequation as follows:

$$
\begin{aligned}
& {\left[U_{i j}(k)\right]\left\{t_{j}\right\}=0,} \\
& {\left[\bar{L}_{i j}(k)\right]\left\{t_{j}\right\}=0,}
\end{aligned}
$$

where $t=\partial \phi / \partial n_{s},\left\{t_{j}\right\}$ is the boundary mode and the wave number, $k$, is embedded in each element of the matrices, $[U]$ and $[\bar{L}]$.

After combining the dual equations on the degenerate boundary when $x$ collocates on $C^{+}$or $C^{-}$, the nontrivial eigensolution exists only when the determinant of the influence matrix is zero by the direct search method. Since either one of the two equations, $U T$ or $L M$, for the outer boundary $S$ can be selected, two alternative approaches, $U T+L M$ and $L M+U T$, are proposed as follows:

The $U T+L M$ method has the eigenequation

$$
\left[K_{\mathrm{UL}}\right]\{\underset{\sim}{t}\}=\left[\begin{array}{rrr}
U_{i_{S} j_{S}} & U_{i_{S} j_{C^{+}}} & U_{i_{S} j_{C^{-}}} \\
U_{i_{C^{+}} j_{S}} & U_{i_{C^{+}} j_{C^{+}}} & U_{i_{C+} j_{C^{-}}} \\
L_{i_{C^{+}} j_{S}} & L_{i_{C^{+}} j_{C^{+}}} & L_{i_{C^{+}} j_{C^{-}}}
\end{array}\right]\left\{\begin{array}{c}
t_{j_{S}} \\
t_{j_{C^{+}}} \\
t_{j_{C^{-}}}
\end{array}\right\}=0,
$$

where $i_{S}$ and $i_{C^{+}}$denote the collocation points on the $S$ and $C^{+}$boundaries, respectively, and $j_{S}$ and $j_{C^{+}}$denote the element ID on the $S$ and $C^{+}$boundaries, respectively.

The $L M+U T$ method has the eigenequation

$$
\left[K_{\mathrm{LU}}\right]\{t\}=\left[\begin{array}{rrr}
L_{i_{S} j_{S}} & L_{i_{S} j_{C^{+}}} & L_{i_{S} j_{C^{-}}} \\
L_{i_{C^{+}} j_{S}} & L_{i_{C^{+}} j_{C^{+}}} & L_{i_{C^{+} j_{C^{-}}}} \\
U_{i_{C^{+}} j_{S}} & U_{i_{C^{+}} j_{C^{+}}} & U_{i_{C^{+} j_{C^{-}}}}
\end{array}\right]\left\{\begin{array}{c}
t_{j_{S}} \\
t_{j_{C^{+}}} \\
t_{j_{C^{-}}}
\end{array}\right\}=0 .
$$

To solve for the eigenequation, a direct search method is employed. 


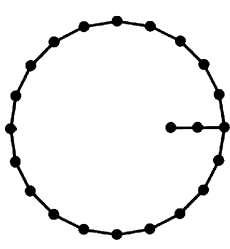

(a)

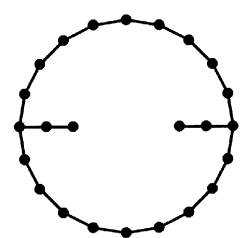

(b)

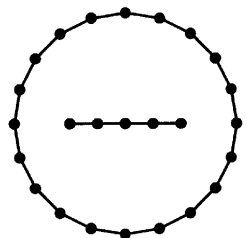

(c)

Fig. 2. The boundary element mesh: (a) single edge stringer, (b) two opposite edge stringers, (c) an internal stringer.

\section{Dynamic stress intensity factor for the membrane with stringers}

For the membrane with stringers shown in Fig. 1(a)-(c), we have the nonhomogeneous boundary conditions as follows:

$$
\begin{aligned}
& \left.\phi(r, \theta)\right|_{r=b}=\sin (\theta / 2), \quad \text { for Fig.1(a), } \\
& \left.\phi(r, \theta)\right|_{r=b}=\sin ^{2}(\theta), \quad \text { for Fig.1(b), } \\
& \left.\phi(r, \theta)\right|_{r=b}=\sin ^{2}(\theta), \quad \text { for Fig.1(c), } \\
& \phi(r, \theta)=0, \quad(r, \theta) \text { on the stringer. }
\end{aligned}
$$

After considering the behavior at the stringer tip, the dynamic stress intensity factors, $K_{w}$, can be determined by

$$
K_{w}=\frac{k \sqrt{R / 2 \pi}}{\sin (k R)} \int_{0}^{2 \pi} \sin \left(\frac{\theta^{\prime}}{2}\right) \phi\left(R, \theta^{\prime}\right) \mathrm{d} \theta^{\prime},
$$

where $R$ is the radius of a circle enclosing the singularity.

\section{Numerical examples}

We next consider the three problems illustrated in Fig. 1(a)-(c), which have been solved by Givoli and Vigdergauz [21]. A circular membrane is given with radius $b$. A single stringer, two opposite edge stringers and an internal stringer are shown in Fig. 1(a)-(c), respectively. The length of the stringers is $a$, as shown. For simplicity, we set $b=1$. The length of the stringer varies from 0 to 1 with an increment of 0.2 . For the special cases, $a=0$ and $a=2$ in Fig. 1(a) and (c) and $a=1$ in Fig. 1(b), we have the extreme cases of no stringer in a circular and a half circular membrane. For any value of $0 \leq a \leq 1$ other than $0,0.5$ and 1.0 , the solution must be obtained numerically. In this study, the dual BEM was employed and the results were compared with the exact solution, the DtN-FEM data and the ABAQUS results. Two kinds of problems, an eigenproblem for homogeneous boundary conditions and problems related to dynamic stress intensity factors with nonhomogeneous boundary conditions, are considered in the following.

\subsection{Critical wave number}

The boundary element meshes for a single edge stringer and two edge stringers are shown in Fig. 2(a)-(c). The critical wave numbers for the single edge stringer with length $a=1$ can be determined using the direct-search method as shown in Fig. 3(a)-(d). Fig. 3(a) and (b) show a failure to determine the eigenvalue since either the $U T$ or $L M$ equation alone can not solve the problem with a degenerate boundary. After combining the dual equations, Fig. $3(\mathrm{c})$ and (d) show in the dotted lines the local minimum of the first three critical wave numbers obtained using the 


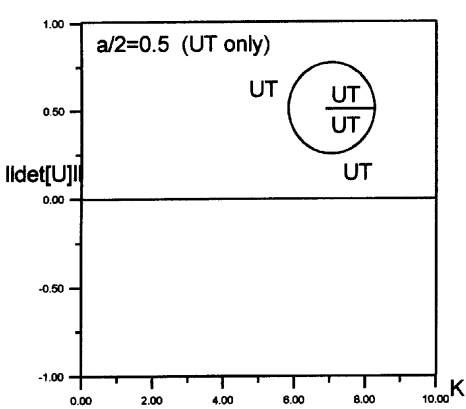

(a)

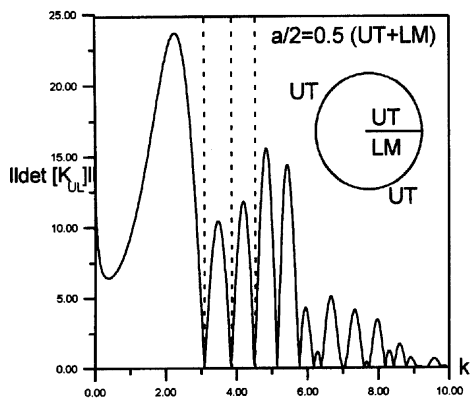

(c)

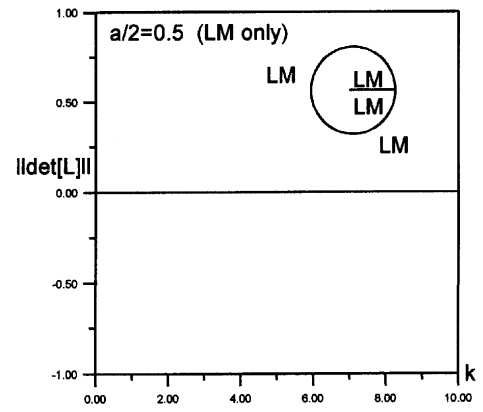

(b)

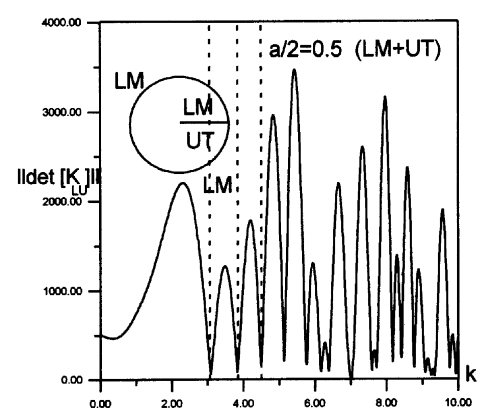

(d)

Fig. 3. The determinant vs. the wave number obtained using (a) the conventional BEM using only the $U T$ equation, (b) the conventional BEM using only the $L M$ equation, (c) the dual BEM using the $U T$ equation (combined with the $L M$ equation), (d) the dual BEM using the $L M$ equation(combined with the $U T$ equation).

Table 2

The first five critical wave numbers $\left(k^{2}\right)$, of a single edge stringer of $a=1$ using DtN-FEM, dual BEM and ABAQUS

\begin{tabular}{llllll}
\hline & Mode 1 & Mode $2\left(J_{1}(3.83 r)\right)$ & Mode 3 & Mode $4\left(J_{2}(5.14 r)\right)$ & Mode 5 \\
\hline DtN-FEM & 9.88 & NA & 14.72 & 20.22 & NA \\
Dual BEM & 9.80 & 14.63 & 20.23 & 26.43 & 33.29 \\
ABAQUS & 9.91 & 14.68 & 20.09 & 26.20 & 32.95 \\
Exact Sol. & 9.87 & 20.19 & 33.32 \\
\hline
\end{tabular}



direct-search method. To check the accuracy of the method, we compared the numerical solution with the analytical solution for the case of $a=1.0$. Also, finite element solutions obtained using ABAQUS were used to double check the comparison. Table 2 shows the first five modes for the single edge stringer with length $a=1 \mathrm{~m}$, using DtN-FEM, the dual BEM and ABAQUS. The mode shapes are shown in Fig. 4 (a)-(e) and Fig. 5(a)-(e) using the dual BEM and ABAQUS, respectively. It is interesting to find that both the dual BEM and the ABAQUS program obtain the two modes of $J_{1}(3.83 r)$ and $J_{2}(5.14 r)$. The two modes both have nodal lines across the stringer as shown in Fig. 4(b) and (d) and Fig. 5(b) and (d); however, the two modes are not found when the DtN-FEM method was employed [21]. The reason is that only the symmetric case in the DtN-FEM formulation is considered in the half model. Nevertheless, the two modes are also eigensolutions since they satisfy the governing equation and boundary conditions. Table 3 shows the squares of the first three critical wave numbers, $k^{2}$, of the single edge stringer for various stringer lengths of $0<a<1$ with an increment of 0.2 . Table 4 shows the squares of the first two critical wave numbers, $k^{2}$, of the two edge stringers for various stringer lengths. It is also found that solutions for the cases 


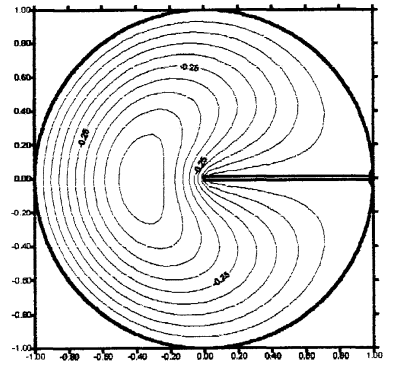

(a) $k=3.13$

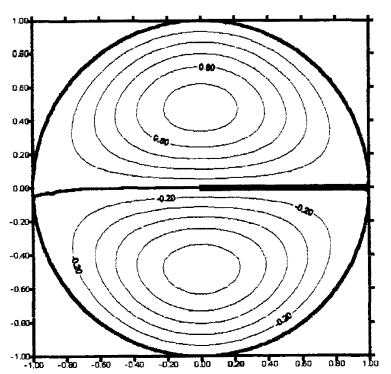

(b) $k=3.83$

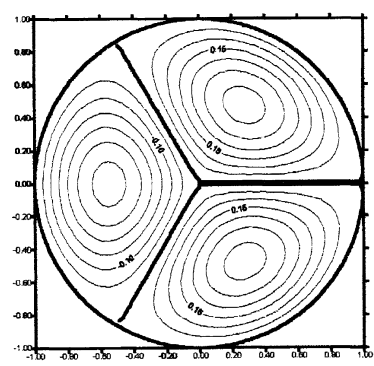

(c) $k=4.49$



(d) $k=5.14$

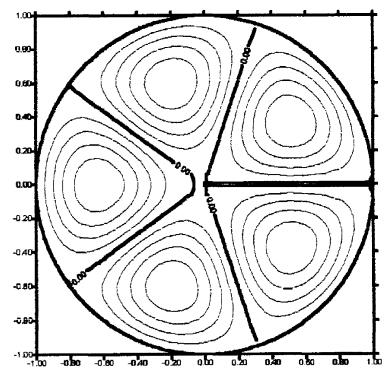

(e) $k=5.75$

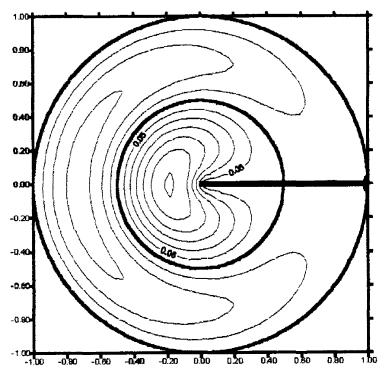

(f) $k=6.29$

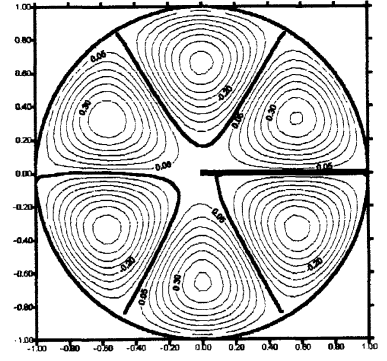

(g) $k=6.36$

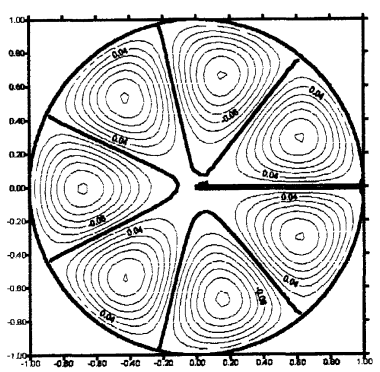

(h) $k=6.96$

Fig. 4. The first eight modes of the single stringer with $a=1$ obtained using the dual BEM: (a) the first mode, (b) the second mode $\left(J_{1}(3.83 r)\right)$, (c) the third mode, (d) the fourth mode $\left(J_{2}(5.14 r)\right)$, (e) the fifth mode, (f) the sixth mode, (g) the seventh mode, (h) the eighth mode. 




(a) $k=3.14$

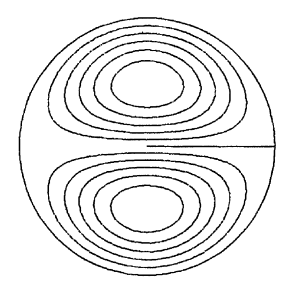

(b) $k=3.82$

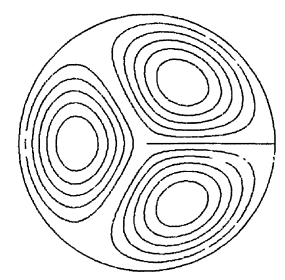

(c) $\mathrm{k}=4.48$

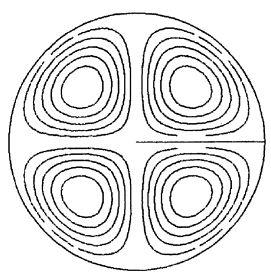

(d) $\mathrm{k}=5.12$

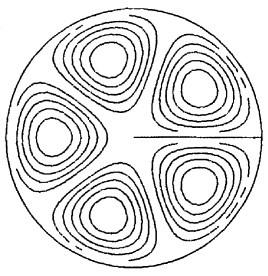

(e) $k=5.74$

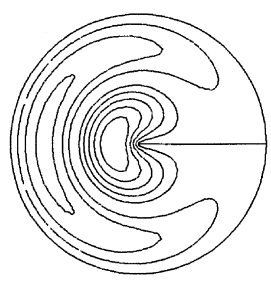

(f) $k=6.27$

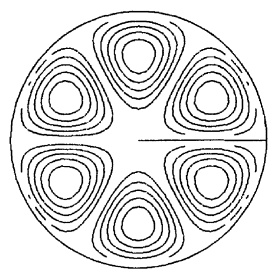

(g) $k=6.35$

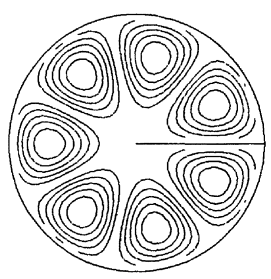

(h) $k=6.95$

Fig. 5. The first eight modes of the single stringer configuration, with $a=1$ obtained using ABAQUS: (a) the first mode, (b) the second mode $\left(J_{1}(3.83 r)\right)$, (c) the third mode, (d) the fourth mode $\left(J_{2}(5.14 r)\right)$, (e) the fifth mode, (f) the sixth mode, (g) the seventh mode, (h) the eighth mode.

of no stringer with $a=0$ and $a=2$ are not obtainable using the DtN-FEM method since it embedes a tip of the stringer in the formulation. For the case of two edge stringers with $a=0.5$, the first eight modes obtained using the dual BEM and ABAQUS, respectively are shown in Figs. 6 and 7. The first six critical wave numbers (squares) are summarized in Table 5. It is interesting to find that all six modes can be obtained using the dual BEM and the ABAQUS program. However, the four modes, including the second $\left(J_{1}(3.83 r)\right)$ the third, the fourth $\left(J_{2}(5.14 r)\right)$ and the fifth modes, are not available for DtN-FEM. The reason why some modes are not available for the four modes shown in Fig. 6(b)-(d) and (f) obtained using the dual BEM or in Fig. 7(b)-(f) obtained using the ABAQUS program is that only symmetry boundary conditions for the quarter model are assumed.

Dynamic stress intensity factor:

Figs. 8-10 are the contour plots of the displacement obtained using the dual BEM for the single edge stringer, the two edge stringers and the internal stringer, respectively. The results compare well with those obtained using DtN-FEM. Also, the exact solution for the dynamic stress intensity factor for the single edge stringer with length 




(a) $k=2.79$



(b) $k=3.83$

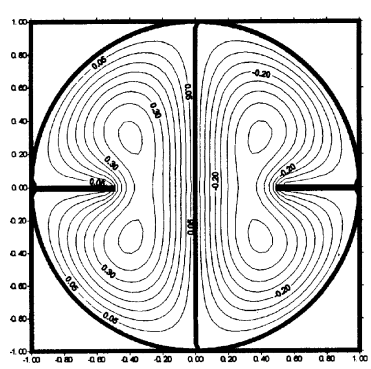

(c) $k=4.66$



(d) $k=5.14$

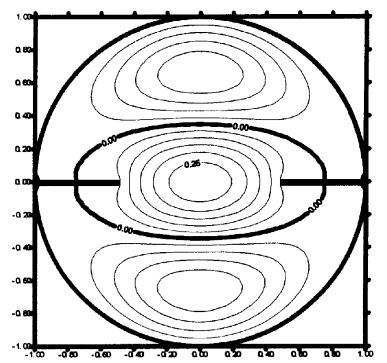

(e) $k=5.44$

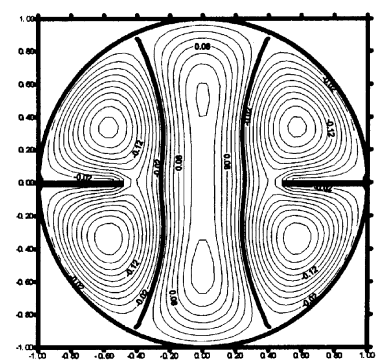

(f) $k=6.28$

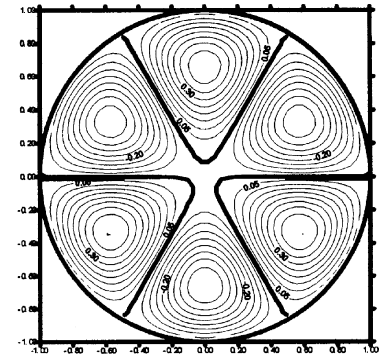

(g) $k=6.38$

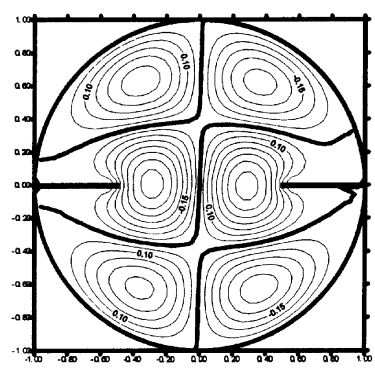

(h) $k=6.97$

Fig. 6. The first eight modes of the two stringer configuration, with $a=0.5$ obtained using the dual BEM: (a) the first mode, (b) the second mode $\left(J_{1}(3.83 r)\right)$, (c) the third mode, (d) the fourth mode $\left(J_{2}(5.14 r)\right)$, (e) the fifth mode, (f) the sixth mode, $(\mathrm{g})$ the seventh mode, (h) the eighth mode. 


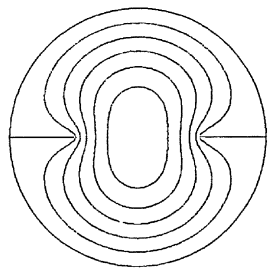

(a) $\mathrm{k}=2.82$

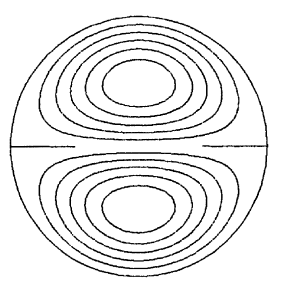

(b) $k=3.83$



(c) $\mathrm{k}=4.67$



(d) $\mathrm{k}=5.12$

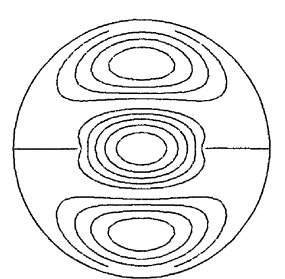

(e) $k=5.43$



(f) $\mathrm{k}=6.26$

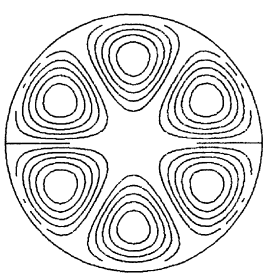

(g) $\mathrm{k}=6.35$

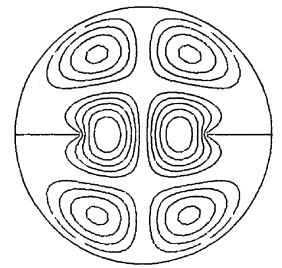

(h) $k=6.96$

Fig. 7. The first eight modes of the two stringer configuration, with $a=0.5$ obtained using ABAQUS: (a) the first mode, (b) the second mode $\left(J_{1}(3.83 r)\right)$, (c) the third mode, (d) the fourth mode $\left(J_{2}(5.14 r)\right)$, (e) the fifth mode, (f) the sixth mode, (g) the seventh mode, (h) the eighth mode.

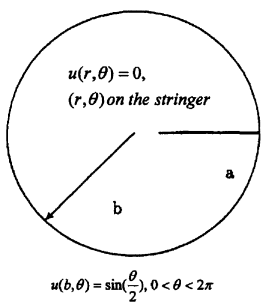

(a)

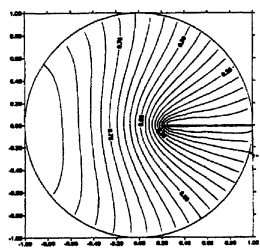

(b)

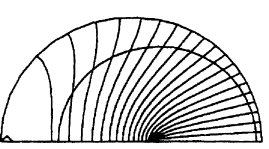

(c)

Fig. 8. (a) Single edge stringer configuration, with $a=0.8$ and $k=1$. (b) Contours of the displacement for the single edge stringer configuration, with $a=0.8$ and $k=1$ obtained using the dual BEM. (c) Contours of the displacement for the single edge stringer configuration, with $a=0.8$ and $k=1$ obtained using DtN-FEM [21]. 
Table 3

The first four critical wave numbers (squares), $k^{2}$, of the exact solution, dual BEM (present), DtN-FEM and FEM obtained using ABAQUS for the membrane problem with an edge stringer

\begin{tabular}{|c|c|c|c|c|c|c|c|c|c|c|c|c|}
\hline & Method & String & ngth $a$ & & & & & & & & & \\
\hline & & 0 & 0.1 & 0.2 & 0.3 & 0.4 & 0.5 & 0.6 & 0.7 & 0.8 & 0.9 & 1.0 \\
\hline \multirow[t]{4}{*}{ Mode 1} & DtN-FEM & NA & 6.03 & 6.41 & 7.02 & 8.23 & 9.88 & 12.09 & 14.03 & 14.81 & 15.08 & NA \\
\hline & Dual BEM & 5.78 & 5.91 & 6.30 & 7.02 & 8.15 & 9.80 & 11.99 & 13.92 & 14.65 & 14.71 & 14.71 \\
\hline & ABAQUS & 5.78 & & & & & 9.91 & & & & & 14.63 \\
\hline & Exact Sol. & 5.78 & & & & & 9.87 & & & & & 14.68 \\
\hline \multirow[t]{4}{*}{ Mode 2} & DtN-FEM $^{\mathrm{a}}$ & NA & 15.83 & 17.11 & 18.83 & 20.04 & 20.22 & 20.60 & 22.95 & 26.30 & 27.63 & NA \\
\hline & Dual BEM & 14.71 & 14.72 & 14.72 & 14.72 & 14.72 & 14.72 & 14.71 & 14.71 & 25.68 & 26.41 & 26.43 \\
\hline & ABAQUS & 14.64 & & & & & 14.63 & & & & & 26.21 \\
\hline & Exact Sol. & 14.68 & & & & & 14.68 & & & & & 26.37 \\
\hline \multirow[t]{4}{*}{ Mode 3} & DtN-FEM & NA & 28.66 & 29.39 & 30.50 & 33.15 & 33.32 & 33.52 & 34.69 & 40.09 & 43.81 & NA \\
\hline & Dual BEM & 26.43 & 15.22 & 16.69 & 18.57 & 19.94 & 20.23 & 20.50 & 22.65 & 26.41 & 40.73 & 40.80 \\
\hline & ABAQUS & 26.21 & & & & & 20.09 & & & & & 40.29 \\
\hline & Exact Sol. & 26.37 & & & & & 20.19 & & & & & 40.71 \\
\hline \multirow[t]{4}{*}{ Mode 4} & DtN-FEM & NA & & & & & & & & & & NA \\
\hline & Dual BEM & 30.49 & 26.45 & 26.44 & 26.44 & 26.43 & 26.43 & 26.43 & 26.43 & 38.28 & 49.32 & 49.33 \\
\hline & ABAQUS & 30.24 & & & & & 26.20 & & & & & 48.61 \\
\hline & Exact Sol. & 30.47 & & & & & 26.37 & & & & & \\
\hline
\end{tabular}

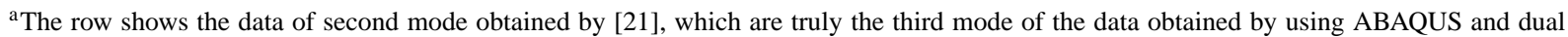
BEM, since the mode (e.g., $J_{1}(3.83 r)$ ) is not available.

Table 4

The first three critical wave numbers (square), $k^{2}$, of exact solution, dual BEM (present method), DtN-FEM and FEM obtained using ABAQUS for the membrane problem with two edge stringers



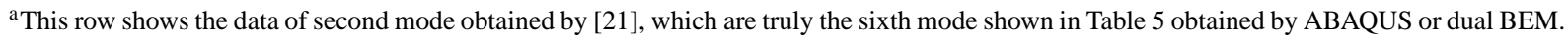

$a=1.0$ is compared with that obtained using the dual BEM. According to Eq. (34), the dynamic stress intensity factor for the edge stringer problem with $a=1.0, b=1.0$ and $k=1.0$ obtained by the dual BEM is (1.50). The result is satisfactory after comparing with the exact solution (1.49) and that of DtN-FEM (1.49). The results of two additional examples are shown in Table 6 . The present method is accurate for the problems that have been considered. 
Table 5

The first six critical wave numbers (square), $k^{2}$, for the case of two edge stringers with $a=0.5$ using DtN-FEM, dual BEM and ABAQUS ${ }^{\mathrm{a}}$

\begin{tabular}{lllllll}
\hline Method & Mode 1 & Mode $2\left(J_{1}(3.83 r)\right)$ & Mode 3 & Mode $4\left(J_{2}(5.14 r)\right)$ & Mode 5 & Mode 6 \\
\hline DtN-FEM & 7.91 & NA & NA & NA & NA & 39.01 \\
Dual BEM & 7.82 & 14.71 & 21.76 & 26.44 & 29.65 & 39.50 \\
ABAQUS & 7.94 & 14.64 & 21.96 & 26.21 & 29.48 & 39.24 \\
Exact Sol. & & 14.68 & & 26.41 & & \\
\hline
\end{tabular}



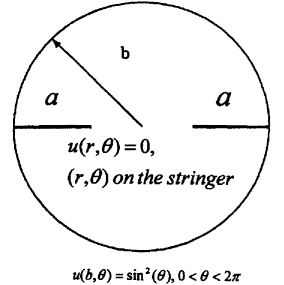

(a)

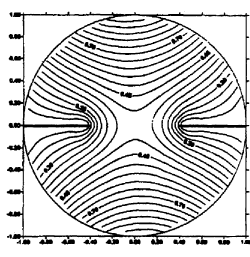

(b)

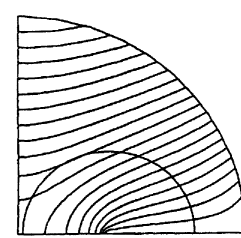

(c)

Fig. 9. (a) Two edge stringer configuration, with $a=0.6$ and $k=1$. (b) Contours of the displacement for the two edge stringer configuration, with $a=0.6$ and $k=1$ obtained using the dual BEM. (c) Contours of the displacement for the two edge stringer configuration, with $a=0.6$ and $k=1$ obtained using DtN-FEM [21].



(a)

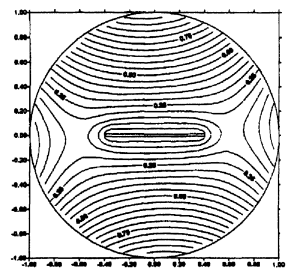

(b)



(c)

Fig. 10. (a) Internal stringer with $a=0.8$ and $k=1$. (b) Contours of the displacement for the internal stringer with $a=0.8$ and $k=1$ obtained using the dual BEM. (c) Contours of the displacement for the internal stringer with $a=0.8$ and $k=1$ obtained using DtN-FEM [21].

Table 6

The single edge stringer problem: stress intensity factors $(\boldsymbol{K})$ for three stringers with different lengths $\left(k^{2}=1\right)$

\begin{tabular}{lllll}
\hline$k^{2}$ & Method & 0.4 & 0.5 & 0.6 \\
\hline 1.0 & DtN-FEM & 1.48 & 1.49 & 1.53 \\
& Dual BEM & 1.41 & 1.50 & 1.61 \\
& Exact Sol. & NA & 1.49 & NA
\end{tabular}

\section{Conclusion}

In this paper, we have demonstrated the usefulness of the dual BEM in solving problems which involve wave scattering from stringer-type singularities. We have considered time-harmonic waves around stringers in a membrane. This setup was chosen as a model for studying wave scattering due to geometrical singularities, which is simpler than two-dimensional elasticity.

The general formulation of the dual integral equations of the boundary value problem for the two-dimensional Helmholtz equation with a degenerate boundary has been derived in this paper. A dual BEM program, DUALHAK, 
has been implemented to solve for the critical wave numbers and vibration modes for a membrane with stringers. Also, the dynamic stress intensity factors have been determined using the program. Three example cases have been successfully solved using the proposed dual BEM, and the results compare well with those obtained using other numerical methods and with the exact solution.

\section{Acknowledgements}

Financial support from the National Science Council, under Grant No. NSC-88-2211-E-019-005, for National Taiwan Ocean University is gratefully acknowledged.

\section{References}

[1] J.T. Chen, S.L. Lin, C.Y. Chiou, S.W. Chyuan, J.Y. Hwang, W.R. Harn, W.T. Chin, Finite Element Analysis and Engineering Applications Using MSC/NASTRAN, Northern Gate Publ., Taipei, 1996 (in Chinese).

[2] T.A. Cruse, F. Rizzo, Computational Applications in Applied Mechanics AMD-11 (1975) 31-46.

[3] C. Zhang, J.D. Achenbach, A new boundary integral equation formulation for elastodynamic and elastostatic crack analysis, ASME J. Appl. Mech. 56 (1989) 284-290.

[4] H.-K. Hong, J.T. Chen, Derivation of integral equations in elasticity, J. Eng. Mech. Div. ASCE, 114(6) EM5 (1988) 1028-1044.

[5] J.T. Chen, H.-K. Hong, S.W. Chyuan, Boundary element analysis and design in seepage flow problems with sheetpiles, Finite Elements in Analysis and Design 17 (1994) 1-20.

[6] C.S. Wang, S. Chu, J.T. Chen, Boundary element method for predicting store airloads during its carriage and separation procedures, in: S. Grilli, C.A. Brebbia, A.H.D. Cheng (Eds.), Computational Engineering with Boundary Elements, Vol. 1: Fluid and Potential Problems, 1990, pp. 305-317.

[7] J.T. Chen, H.-K. Hong, Boundary Element Method, 2nd ed., New World Press, Taipei, Taiwan, 1992 (in Chinese).

[8] J.T. Chen, K.H. Chen, W. Yeih, N.C. Shieh, Dual boundary element analysis for cracked bars under torsion, Eng. Computations 15(6) (1998) 732-749.

[9] J.T. Chen, K.H. Chen, Dual integral formulation for determining the acoustic modes of a two-dimensional cavity with a degenerate boundary, Eng. Anal. with Boundary Elements 21 (1998) 105-116.

[10] J.T. Chen, K.H. Chen, S.W. Chyuan, 1998, Numerical experiments for acoustic modes of a square cavity using the dual BEM, Appl. Acoustics 57 (1999) 293-325.

[11] K.H. Chen, J.T. Chen, D.Y. Liou, Dual boundary element analysis for a acoustic cavity with an incomplete partition, Chinese J. Mech. 14(2) (1998) 1-11.

[12] J.T. Chen, F.C. Wong, Dual formulation of multiple reciprocity method for the acoustic mode of a cavity with a thin partition, J. Sound Vibration 217(1) (1998) 75-95.

[13] J.T. Chen, H.-K. Hong, Review of Dual Boundary Element Method with Emphasis on Hypersingular Integrals and Divergent Series, Appl. Mech. Rev. ASME 52(1) (1998) 17-33.

[14] A.J. Burton, G.F. Miller, The application of integral equation methods to numerical solution of some exterior boundary value problems, Proc. R. Soc. London Ser A 323 (1971) 201.

[15] P. Fedelinski, M.H. Aliabadi, D.P. Rooke, The dual boundary element method in dynamic fracture mechanics, Eng. Anal. Bound. Elem. 12 (1993) 203-210.

[16] P. Fedelinski, M.H. Aliabadi, D.P. Rooke, The dual boundary element method: J-integral for dynamic stress intensity factors, Int. J. Fract. 65 (1994) 369-381.

[17] P. Fedelinski, M.H. Aliabadi, D.P. Rooke, A single-region time-domain BEM for dynamic crack problem, Int. J. Solids Struct. 32 (1995) 3555-3571.

[18] P. Fedelinski, M.H. Aliabadi, D.P. Rooke, Boundary element formulations for the dynamic analysis of cracked structures, Eng Anal. Bound. Elem. 17 (1996) 45-56.

[19] G.E. Blandford, A.R. Ingraffea, J.A. Liggett, Two-dimensional stress intensity factor computations using the boundary element method, Int. J. Numer. Meth. Eng. 17 (1981) 387-404.

[20] O.E. Lafe, J.S. Montes, A.H.D. Cheng, J.A. Liggett, P.L-F. Liu, Singularity in Darcy flow through porous media, J. Hydraulics Division, ASCE 106 HY6 (1980) 977-997.

[21] D. Givoli, S. Vigdergauz, Finite element analysis of wave scattering from singularities, Wave Motion 20 (1994) 165-176. 